Chirurg 2017·88:972

https://doi.org/10.1007/s00104-017-0519-8

Online publiziert: 30. Oktober 2017

๑) Springer Medizin Verlag GmbH 2017

CrossMark

M. Kueckelhaus ${ }^{1} \cdot$ T. Hirsch ${ }^{1}$ M. Lehnhardt ${ }^{1}$ A. Daigeler ${ }^{1,2}$

'Klinik für Plastische Chirurgie und Schwerbrandverletzte, Handchirurgie-Zentrum, operatives Referenzzentrum für Gliedmaßentumoren, Berufsgenossenschaftliches Universitätsklinikum Bergmannsheil, Ruhr-Universität Bochum, Bochum, Deutschland

${ }^{2}$ Klinik für Hand-, Plastische, Rekonstruktive und Verbrennungschirurgie , BG Klinik Tübingen , Tübingen, Deutschland

\title{
Erratum zu: Nekrotisierende Fasziitis der oberen und unteren Extremität
}

\section{Erratum zu:}

Chirurg 2017

https://doi.org/10.1007/s00104-017-

0397-0

Die erste Empfehlung im Abschnitt „Therapie - Antibiotische Behandlung" ist missverständlich ausgedrückt. Es handelt sich bei der Breitspektrumantibiose um eine Kombination der genannten Antibiotika. Es sollte daher besser heißen:

Bei Verdacht auf eine NF sollte neben der chirurgischen Therapie unverzüglich eine kalkulierte i. v. antibiotische Behandlung zur Abdeckung eines breiten Spektrums gestartet werden.

In $\bullet$ Tab. 3 dieses Beitrags wurden einige Einheiten nicht korrekt angegeben. Die korrigierte Tabelle können Sie nachfolgend entnehmen.

Bitte beachten Sie zudem die korrekte Nennung der Autorenschaft: M. Kueckelhaus, T. Hirsch, M. Lehnhardt und A. Daigeler.

\section{Korrespondenzadresse}

Prof. Dr. A. Daigeler

Klinik für Hand-, Plastische, Rekonstruktive und Verbrennungschirurgie, BG Klinik Tübingen Schnarrenbergstr. 95, 72076 Tübingen, Deutschland adaigeler@bgu-tuebingen.de
Tab. 3 LRINEC-Score zur Frühdiagnostik der nekrotisierenden Fasziitis

\begin{tabular}{|c|c|}
\hline Laborwert & Punktzahl \\
\hline \multicolumn{2}{|c|}{ C-reaktives Protein (mg/l) } \\
\hline$\leq 150$ & 0 \\
\hline$>150$ & 4 \\
\hline \multicolumn{2}{|l|}{ Leukozyten (/nl) } \\
\hline$<15$ & 0 \\
\hline $15-25$ & 1 \\
\hline$>25$ & 2 \\
\hline \multicolumn{2}{|c|}{ Hämoglobin (g/dl) } \\
\hline$>13,5$ & 0 \\
\hline $11-13,5$ & 1 \\
\hline$<11$ & 2 \\
\hline \multicolumn{2}{|l|}{ Natrium (mmol/l) } \\
\hline$\geq 135$ & 0 \\
\hline$<135$ & 2 \\
\hline \multicolumn{2}{|l|}{ Kreatinin (mg/dl) } \\
\hline$\leq 1,6$ & 0 \\
\hline$>1,6$ & 2 \\
\hline \multicolumn{2}{|l|}{ Glukose (mg/dl) } \\
\hline$\leq 180$ & 0 \\
\hline$>180$ & 1 \\
\hline \multicolumn{2}{|l|}{ Risikokategorien $^{\mathrm{a}}$} \\
\hline Niedrig $(<50 \%)$ & LINREC $\leq 5$ Punkte \\
\hline Mittel (50-75 \%) & LINREC 6-7 Punkte \\
\hline Hoch (>75 \%) & LINREC $\geq 8$ Punkte \\
\hline \multicolumn{2}{|c|}{$\begin{array}{l}\text { a } \%=\text { Wahrscheinlichkeit für das Vorliegen } \\
\text { einer Nekrotisierenden Fasziitis } \\
\text { LRINEC Laboratory Risk Indicator for Necro- } \\
\text { tizing }\end{array}$} \\
\hline
\end{tabular}

Die Online-Version des Originalartikels ist unter https://doi.org/10.1007/s00104-017-0397-0 zu finden. 